\title{
Herpes simplex virus type 2 or human herpesvirus 8 infection and prostate cancer risk: A meta-analysis
}

\author{
XIAOXIAO GE ${ }^{1}$, XIAO WANG ${ }^{2}$ and PENG SHEN ${ }^{1}$ \\ Departments of ${ }^{1}$ Medical Oncology and ${ }^{2}$ Urology, The First Affiliated Hospital, College of Medicine, \\ Zhejiang University, Hangzhou, Zhejiang 310000, P.R. China
}

Received December 20, 2012; Accepted February 11, 2013

DOI: $10.3892 /$ br. 2013.82

\begin{abstract}
Prostate cancer is the second most frequently diagnosed type of cancer and the sixth leading cause of cancer mortality among males worldwide. The aim of this study was to investigate the association between the infection by herpes simplex virus type 2 (HSV-2) or human herpesvirus 8 (HHV-8) and the risk of prostate cancer. A systematic literature search was performed using PubMed, Cochrane Library, Web of Science, Scopus, CNKI and CBM. The association of HSV-2 or HHV-8 infection with the risk of prostate cancer was separately assessed. Estimates of the odds ratio (OR) with $95 \%$ confidence interval (CI) were pooled by the fixed- or random-effects model. A total of 11 articles with 2,996 cases and 3,875 controls were included in this meta-analysis. HSV-2 infection was associated with increased prostate cancer risk $(\mathrm{OR}=1.209$; 95\% CI, 1.003-1.456). Results of the stratified analysis suggested that such an association existed among participants from North and South America (OR=1.226; 95\% CI, 1.000-1.503). No significant correlation was observed in the HHV-8 group $(\mathrm{OR}=1.106 ; 95 \% \mathrm{CI}, 0.765-1.598)$. Further investigations and large-sample studies are required to elucidate the possible mechanism underlying viral carcinogenesis and the association between herpes virus infection and the risk of prostate cancer.
\end{abstract}

\section{Introduction}

Prostate cancer is the second most frequently diagnosed type of cancer and the sixth leading cause of cancer mortality among males worldwide (1). Statistics in the USA showed that 241,740 men were diagnosed with prostate cancer and 28,170 were estimated to succumb to the disease in 2012 (2). Prostate cancer is considered a multifactorial disease. Older age, ethnicity and positive family history are well-established risk factors $(1,3)$.

Correspondence to: Professor Peng Shen, Department of Medical Oncology, The First Affiliated Hospital, College of Medicine, Zhejiang University, 79 Qingchun Road, Hangzhou, Zhejiang 310000, P.R. China

E-mail: zyhlk@sina.cn; shenp@zju.edu.cn

Key words: herpes simplex virus type 2, human herpesvirus 8 , prostatic neoplasms, meta-analysis
Genetic factors were considered important in prostate cancer by several studies (4-7). Since the 1970s, it has been a matter of debate whether prostate cancer, or at least a subgroup of prostate cancer cases, are associated with infection. With the development of serological assays for the detection of infectious agents, studies were conducted to investigate the relationship between prostate cancer and viral infection (8-11).

Herpes virus is one of the viruses most commonly related to carcinogenesis. It was reported that herpes virus plays an important role in the pathogenesis of cancer via the inhibition of cell apoptosis and stimulation of DNA synthesis, which may ultimately lead to cancer (12). Previous studies indicated that infection by herpes simplex virus type 2 (HSV-2) or human herpesvirus 8 (HHV-8) may be associated with a higher prostate cancer risk (12-14). However, other epidemiological studies failed to demonstrate such an association (15-17). Whether HSV-2 or HHV-8 infection is related to prostate cancer remains controversial. In order to further assess the correlation between prostate cancer risk and HSV-2 or HHV-8 infection, a meta-analysis was conducted.

\section{Materials and methods}

Literature search. We performed a systematic literature search using PubMed, Cochrane Library, Web of Science and Scopus in English, as well as CNKI and CBM in Chinese, to identify the publications updated up to May, 2012. The medical subject headings (MeSH) 'Herpesviridae', 'Alphaherpesvirinae', 'Simplexvirus', 'Herpesvirus 2, Human', 'Gammaherpesvirinae', 'Rhadinovirus' and 'Herpesvirus 8, Human' were combined with 'Prostatic Neoplasms'. Common expressions such as 'herpes simplex virus 2', 'human herpesvirus 8', 'prostatic carcinoma' and 'prostate cancer' were also used. In addition, aliases such as 'Kaposi's sarcoma-associated herpesvirus', 'KSHV', 'HHV-8' and 'HSV-2' were all included. Moreover, variant forms of expression were used (presence or absence of a hyphen or space between adjacent words, Roman numerals or English words instead of Arabic numerals and presence or absence of quotation marks). Additional publications were identified from the reference lists of potentially suitable articles and 'related articles' identified during the search.

Inclusion criteria. Each included publication was reviewed to evaluate whether the following criteria were met: i) comparative 
study assessing the association between infection by HSV-2 or HHV-8 and prostate cancer risk; ii) detection method restricted to serological assays; and iii) providing sufficient information to determine herpes virus infection incidence (number of positive vs. negative subjects) in the case and control groups. The inclusion criteria were not restricted by study size, population race or publication language, date or type. For articles with similar population resources or overlapping datasets, only the largest or most recent was included. Studies in which herpes virus was not detected in either group were excluded, since such studies hold no weight in meta-analysis and do not affect conclusions.

Data extraction. Two reviewers independently extracted data from all the potentially qualified articles using a standardized data extraction form, in order to avoid mistakes or omissions. A third reviewer was consulted in the case of discrepancies. Data extracted from the publications included name of the first author, year of publication, number of case and control subjects, exposure frequencies in case and control groups, geographical region and herpes virus subtype.

Statistical analysis. We selected the odds ratio (OR) and 95\% confidence interval (CI) to assess the strength of the association between infection by HSV-2 or HHV-8 and prostate cancer risk. Subgroup analyses were performed according to geographical region (North and South America or Europe). The statistical significance level was set at 0.05. The extent of heterogeneity among included studies was quantified using the Q test (18) and the $\mathrm{I}^{2}$ score (19). In the Q test, $\mathrm{P}<0.05$ was considered to indicate a statistically significant difference. High $\mathrm{I}^{2}$ values reflected increasing heterogeneity. $\mathrm{I}^{2}$ values $<25 \%$ were considered as low and values $<50 \%$ were considered as moderate $(19,20)$. A fixed-effects model with the Mantel-Haenszel method was used to provide a summary estimation of the relationship between herpes virus infection and prostate cancer risk when heterogeneity was not significant (21). Otherwise, the random-effects model was utilized (22). Publication bias was assessed by Egger's (23) and Begg's test (24), with a statistical significance level of 0.05 . The statistical analyses were performed via STATA version 11 (StataCorp, College Station, TX, USA).

\section{Results}

Description of the meta-analysis. We identified a total of 11 articles on infection by HSV-2 or HHV-8 and prostate cancer risk, updated up to May 2012 (8-11,14-17,25-27). The process of identifying and selecting articles is shown in Fig. 1. Four articles were excluded at data extraction, since their results were not considered suitable for this meta-analysis: one of them included a case group only (28), one provided ambiguous information regarding the virus subtype (29), one lacked a number of negative subjects in the case group and of positive subjects in the control group (30) and one adopted a detection method of inadequate specificity for HSV-2 (31).

This meta-analysis consisted of 2,996 cases and 3,875 controls. There were two articles evaluating HSV-2 and HHV-8 in the same (15) or different (11) population groups. One article detecting HHV-8 consisted of two groups of participants from either the Republic of Trinidad and Tobago

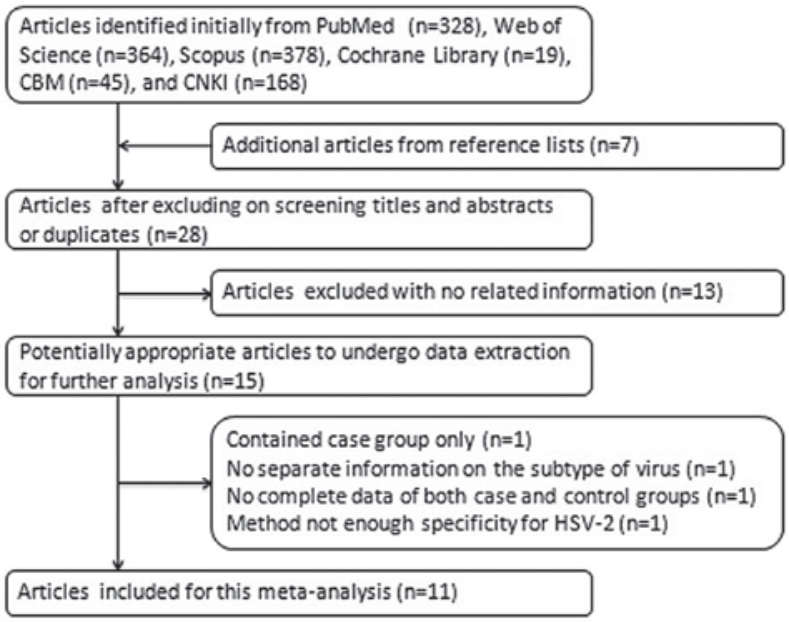

Figure 1. Process of article selection.

or the USA (9), whereas another consisted of four groups from Italy or the USA (16). Studies on different viruses or among separate population groups in the articles mentioned above, were treated as independent studies. Therefore, 19 studies from 11 references were included in this meta-analysis, including 8 on HSV-2 $(10,11,14,15,25-27)$ and 11 on HHV-8 $(8,9,11,15-17)$. Detailed characteristics of the eligible studies are listed in Table I.

$H S V-2$ and prostate cancer risk. Eight studies with 1,721 cases and 2,165 controls were included in this meta-analysis, in order to investigate the association between HSV-2 infection and prostate cancer risk. Five studies were located in North and South America, with 1,213 cases and 1,737 controls $(11,14,25,26)$ and three were located in Europe, with 508 cases and 428 controls $(10,15,27)$. No statistically significant heterogeneity was detected in pooled analysis $\left(\mathrm{P}=0.243, \mathrm{I}^{2}=23.4 \%\right)$ and the fixed-effects model was adopted. A marginally positive relationship was observed between HSV-2 infection and prostate cancer (OR=1.209; 95\% CI, 1.003-1.456) (Fig. 2). No publication bias was found with Begg's or Egger's test ( $\mathrm{P}=0.386$ and $\mathrm{P}=0.291$, respectively). Begg's funnel plot is shown in Fig. 3.

In the stratified analysis by geographical region, no statistically significant heterogeneity was observed in either subgroup (Table II). Therefore, the fixed-effects model was adopted. A marginally positive association between HSV-2 infection and increased prostate cancer risk was demonstrated in the North and South American subgroup (OR=1.226; 95\% CI, 1.000-1.503). However, no statistically positive result was observed in the European subgroup (OR=1.222; 95\% CI, 0.704-1.787). No publication bias was found in the subgroup analyses, as shown in Table II (funnel plot not shown).

HHV-8 and prostate cancer risk. There were 11 studies with 2,306 cases and 3,280 controls evaluating the correlation between HHV-8 infection and prostate cancer risk. There were nine studies in North and South America, with 2,133 cases and 2,958 controls $(8,9,11,16,17)$ and two in Europe, with 173 cases and 322 controls $(15,16)$. The random-effects model 


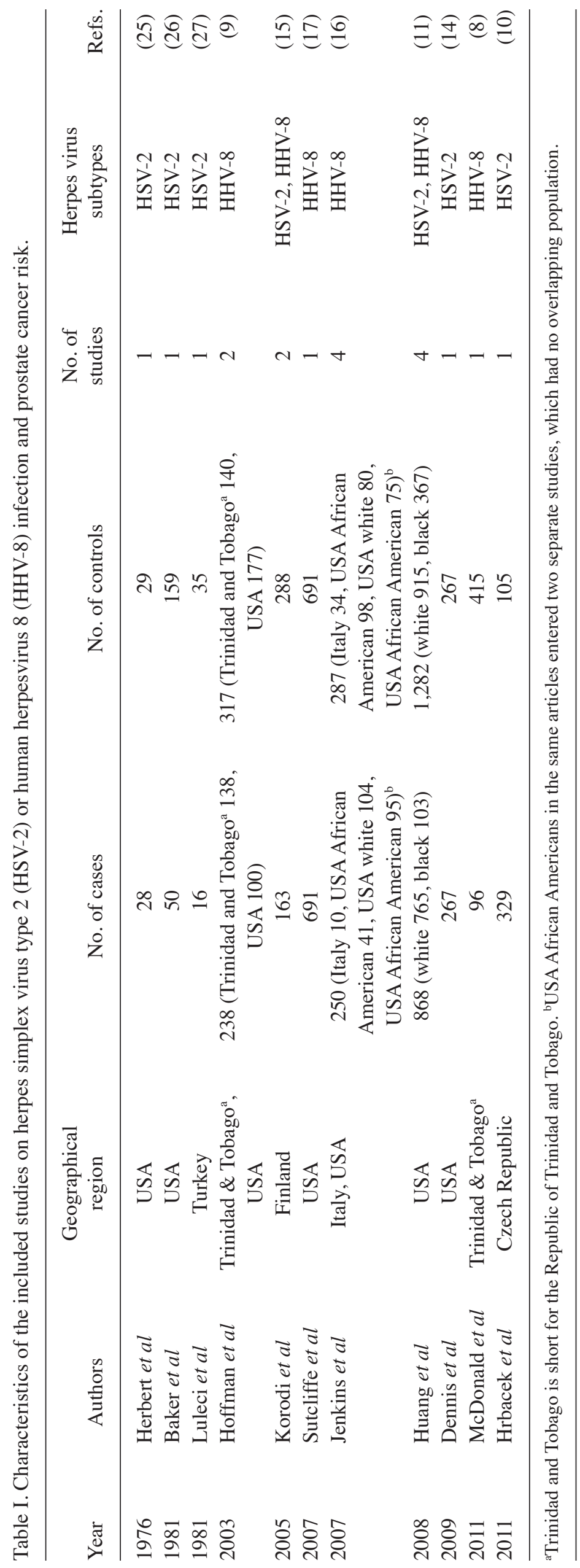


Table II. Stratified pooled OR and 95\% CI for HSV-2 or HHV-8 infection and prostate cancer risk.

\begin{tabular}{|c|c|c|c|c|c|c|}
\hline \multirow[b]{2}{*}{ Subgroup } & \multirow{2}{*}{$\begin{array}{c}\text { No. of } \\
\text { studies (Refs.) }\end{array}$} & \multirow[b]{2}{*}{ OR $(95 \% \mathrm{CI})$} & \multicolumn{2}{|c|}{ Heterogeneity } & \multicolumn{2}{|c|}{ Publication bias P-value } \\
\hline & & & P-value & $\mathrm{I}^{2}(\%)$ & Begg's test & Egger's test \\
\hline \multicolumn{7}{|l|}{ HSV-2 by geographical region } \\
\hline North and South America & $5(11,14,25,26)$ & $1.226(1.000-1.503)$ & 0.192 & 34.4 & 0.624 & 0.284 \\
\hline Europe & $3(10,15,27)$ & $1.122(0.704-1.787)$ & 0.241 & 29.6 & 0.117 & 0.085 \\
\hline \multicolumn{7}{|c|}{ HHV-8 by geographical region } \\
\hline North and South America & $9(8,9,11,16,17)$ & $1.142(0.759-1.718)$ & 0.000 & 78.4 & 0.677 & 0.770 \\
\hline Europe & $2(15,16)$ & $0.839(0.374-1.882)$ & 0.682 & 0.0 & 0.317 & - \\
\hline
\end{tabular}

OR, odds ratio; CI, confidence interval; HSV-2, herpes simplex virus type 2; HHV-8, human herpesvirus 8.

\begin{tabular}{lll} 
HSV-2 & OR (95\% Cl) \\
Study (year) & $1.32(0.43,4.04)$ \\
Herbert (1976) & $2.05(1.05,4.00)$ \\
Baker (1981) & $4.14(0.81,21.17)$ \\
Luleci (1981) & $0.97(0.45,2.08)$ \\
Korodi (2005) & $0.93(0.67,1.30)$ \\
Huang (2008) & $1.19(0.77,1.84)$ \\
Huang (2008) & $1.53(1.03,2.28)$ \\
Dennis (2009) & $0.95(0.50,1.82)$ \\
Hrbacek (2011) & $1.21(1.00,1.46)$ \\
Pooled OR & & \\
\hline
\end{tabular}

Figure 2. Forest plots of herpes simplex virus type 2 (HSV-2) infection and prostate cancer risk for overall analysis. OR, odds ratio; CI, confidence interval.

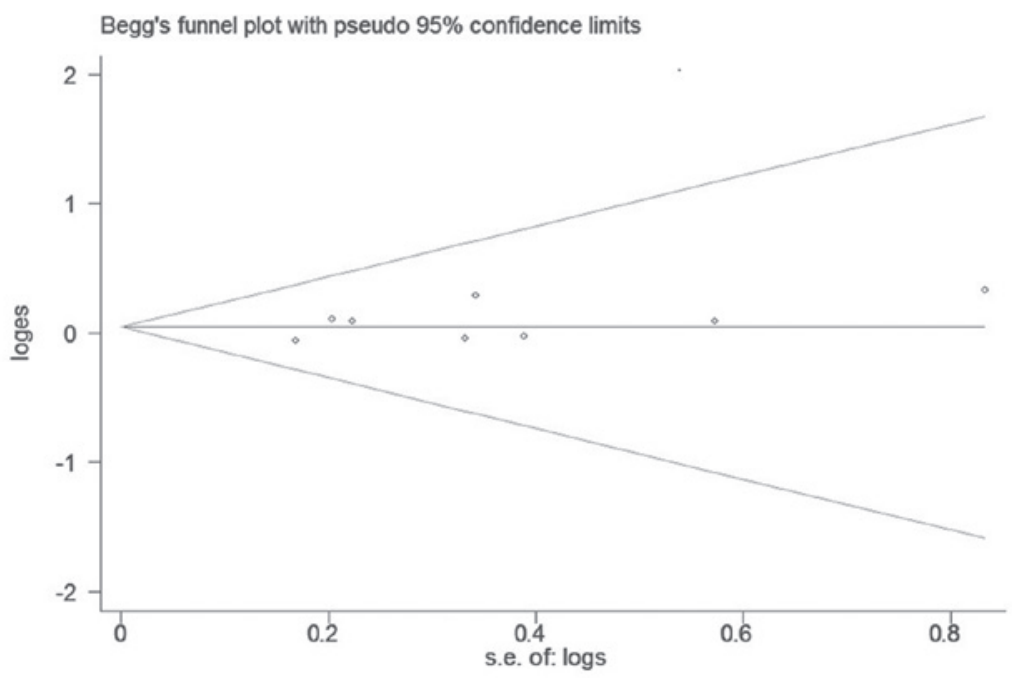

Figure 3. Begg's funnel plots assessing the publication bias on herpes simplex virus type 2 infection and prostate cancer risk. 
HHV-8

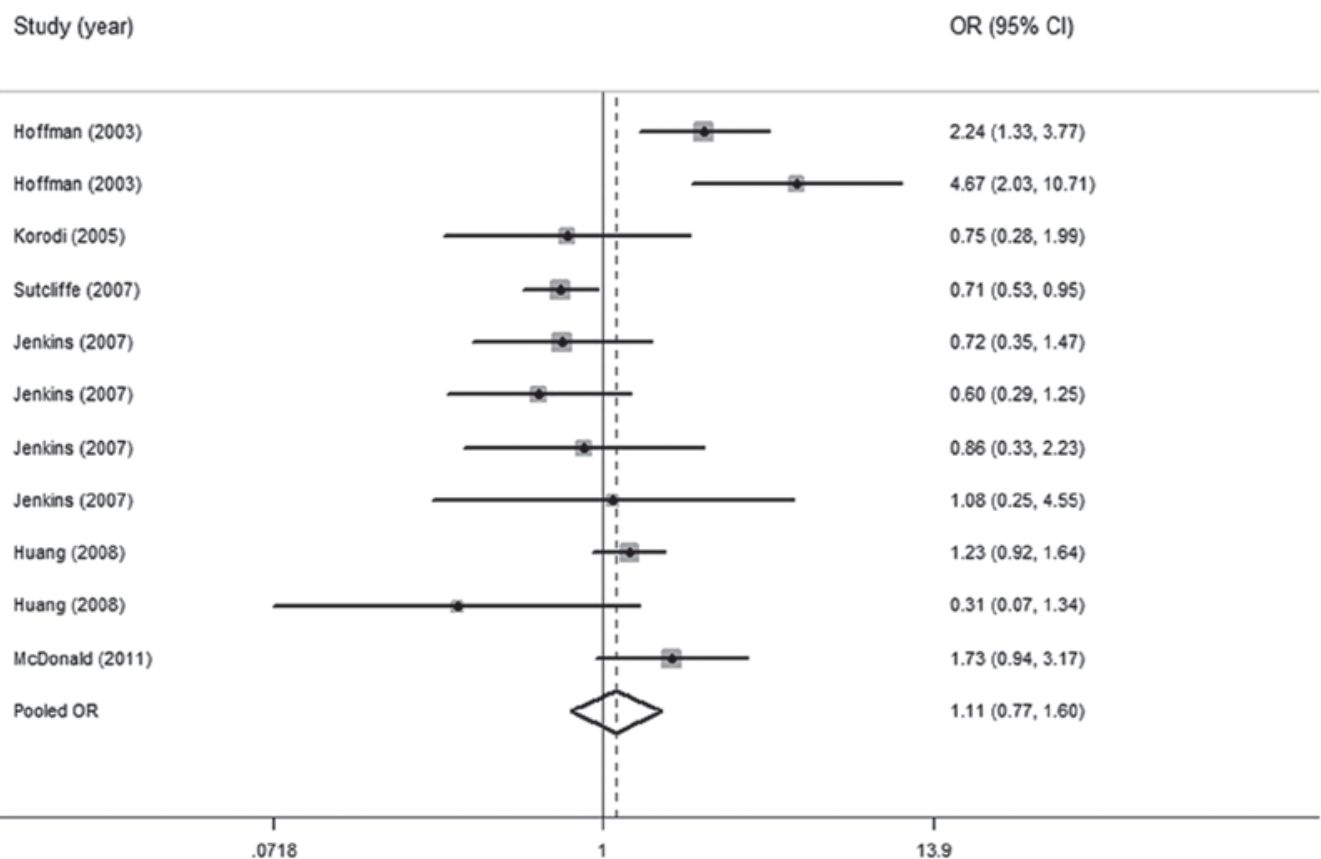

Figure 4. Forest plots of human herpesvirus 8 (HHV-8) infection and prostate cancer risk for overall analysis. OR, odds ratio; CI, confidence interval.

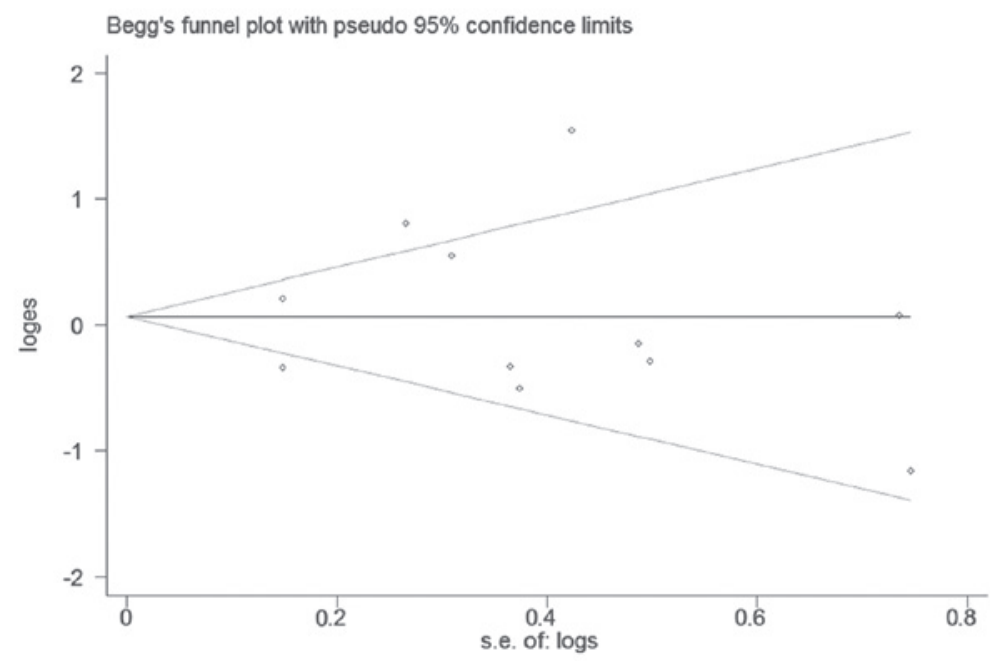

Figure 5. Begg's funnel plots assessing the publication bias on human herpesvirus 8 infection and prostate cancer risk.

was used for summarizing the estimation when heterogeneity was demonstrated by the pooled analysis $(\mathrm{P}=0.000$, $\mathrm{I}^{2}=73.4 \%$ ). No significant relationship was identified between HHV-8 infection and higher prostate cancer risk $(\mathrm{OR}=1.106$; 95\% CI, 0.765-1.598) (Fig. 4).

Stratified analysis was also performed according to geographical region. Heterogeneity was observed in the North and South America subgroup ( $\left.\mathrm{P}=0.000, \mathrm{I}^{2}=78.4 \%\right)$ and the random-effects model was adopted. No association was observed in either subgroup (Table II).

There was no publication bias in the overall (Fig. 5) or stratified analysis (funnel plot not shown). The results of Begg's and Egger's test are presented in Table II.
Sensitivity test. We performed sensitivity analyses by sequentially excluding studies one by one, in order to examine the influence of a single study on the overall estimate or on any stratum. The results demonstrated that no single study considerably affected the summary of risk estimates in this meta-analysis (data not shown).

\section{Discussion}

Viral infection is a potential risk factor for carcinogenesis. Several studies indicated that these infectious agents may elicit an immune response, creating a cytokine tissue environment that leads to chronic inflammation, DNA damage, 
cell proliferation, angiogenesis and ultimately prostate cancer (32-35). An in vitro study suggested that components of viral and other infectious agents may shift the balance towards altered homeostasis in cells that have already deviated from normal gene expression and may thus play a role in malignant transformation (36). Several epidemiological studies evaluated the association between herpes virus infection and prostate cancer risk, although results were inconsistent $(9,14,16,17)$. Therefore, an elaborate and comprehensive demonstration of the association between herpes virus infection and prostate cancer risk is of significance. To the best of our knowledge, this meta-analysis is the first to focus on the relationship between HSV-2 or HHV-8 infection and prostate cancer risk.

Prostate cancer incidence and HSV-2 prevalence are affected by geographical region and age. Prostate cancer incidence rates vary by $>25$-fold worldwide, with the highest rates recorded primarily in the developed countries of Oceania, Europe and North America (1). In a global review, the HSV-2 prevalence is highest in parts of Africa and North and South America, whereas it tends to be lower in Asia (37). In our meta-analysis, the geographical factor was considered and used for study stratification prior to further analysis. However, no studies from Africa or Asia were included, which restricted a thorough comparison among different areas. By contrast, older age is a well-established risk factor for prostate cancer (1). The median age of prostate cancer cases in the USA is 67 years, according to the statistics updated up to 2012 (38). Furthermore, HSV-2 prevalence is also strongly associated with age, increasing from negligible levels in children $<12$ years to as high as $80 \%$ among older age populations (37). We aimed to conduct a stratified analysis according to age. However, of all the studies included, only a few provided the mean or median age $(10,14,26,30)$. Moreover, a study demonstrated that the association with HSV-2 was strengthened when earlier sera analyses were restricted to specimens collected at least five years prior to diagnosis and suggested a long latency period from HSV-2 infection to prostate cancer development (14). We aimed to investigate this association stratified according to the period between serum sample collection and diagnosis. However, due to the insufficient information provided, this investigation could not be conducted.

Some of the studies reported that the HHV-8 DNA sequence was detected in both normal and cancerous prostatic tissues (39-41), whereas others did not (42-45). Moore and Chang (46) reported that HHV-8 expresses viral interleukin-6, a homolog of human interleukin-6, which was suggested to elicit prostate cancer cell proliferation by Platz and De Marzo (35). Several epidemiological studies demonstrated no association between HHV-8 and prostate cancer $(8,11,15,16)$, which is consistent with the results of this meta-analysis (OR=1.106; 95\% CI, 0.765-1.598). Hoffman et al (9) conducted two studies among participants from either the Republic of Trinidad and Tobago or the USA, which demonstrated elevated HHV-8 seropositivity among prostate cancer patients. The consistent results from two independent laboratories with reliable assays and algorithms were the first documented to indicate the positive association. However, not all prostate cancer patients in the two studies were HHV-8 seropositive, suggesting that the virus is not always associated with prostate cancer. Another study included in this meta-analysis indicated an inverse association between HHV-8 infection and prostate cancer risk (17). The confounding factor of Mediterranean heritage in the participants enrolled and the differences in assay sensitivity and specificity were considered by the authors as a possible eplanation of the inverse findings, along with the skewing of the immune response mediated by HHV-8 chemokines, which was hypothesized to exert a potentially protective effect on prostate cancer (46). McDonald et al (8) suggested that HHV-8 might segregate patients with manifest and emergent prostate cancer into two groups, HHV-8 sero-positive prostate cancer detected immediately and HHV-8 sero-negative prostate cancer detected later, which may explain the opposite positive and negative HHV-8 associations observed.

Previous studies detected herpes virus DNA $(41,47)$ or anti-virus antibody in tissue samples (13). However, herpes virus detection in tissues was not frequently performed, since blood samples were easier to collect from the participants compared to tissue samples and the serological assays were more convenient to conduct compared to DNA detection. Thus, serological assays were widely used in studies, in order to guarantee an adequate study size and ensure data reliability to a certain extent. There is no evidence strong enough at present to support that the results of viral infection from blood samples were equal to or comparable with those from tissue samples, therefore, the detection methods for HSV-2 and HHV-8 in this meta-analysis were restricted to serological assays.

The pooled ORs were estimated based on all the studies obtained from systematic search, providing a relatively high statistical power. However, certain limitations should be considered. Firstly, this being a meta-analysis, no original data were obtained. Since prostate cancer is a multifactorial disease and herpes virus infection is related to individual behavior, several covariates, including age, sexual behavior, smoking status and ethnicity, should be taken into consideration to make this meta-analysis more reliable. However, such information could not be summarized without the original data of the recruited studies, which made it difficult to further assess potential confounding factors. Secondly, although different databases were used to cover publications from different areas, there existed a distinction between study quantity and size among different geographical groups, which may bias the conclusions. No studies from Africa or Asia were included; therefore, assessment regarding these areas was not possible. Furthermore, in HSV-2-related analyses, there were five studies with 1,213 cases and 1,737 controls in the North and South American group $(11,14,25,26)$ and three studies with 508 cases and 428 controls in the European group $(10,15,27)$. With fewer studies and participants, the results from the European group may not be representative or stable. Thirdly, the selected databases covered parts of unpublished articles; however, we did not specifically search for these gray data, although no publication bias was observed by tests.

In conclusion, the meta-analysis demonstrated a potential association between HSV-2 infection and a higher prostate cancer risk, confined to the North and South American group, as determined by stratified analysis. An association between HHV-8 infection and prostate cancer was not detected. The results of this study failed to explain the roles that HSV-2 or 
HHV-8 infection may play in prostate cancer. Further investigations and large-sample studies are required to elucidate the potential mechanism underlying viral carcinogenesis and the relationship between herpes virus infection and prostate cancer risk.

\section{References}

1. Jemal A, Bray F, Center MM, Ferlay J, Ward E and Forman D Global cancer statistics. CA Cancer J Clin 61: 69-90, 2011.

2. Siegel R, Naishadham D and Jemal A: Cancer statistics, 2012. CA Cancer J Clin 62: 10-29, 2012.

3. Gunderson K, Wang CY and Wang R: Global prostate cancer incidence and the migration, settlement, and admixture history of the Northern Europeans. Cancer Epidemiol 35: 320-327, 2011

4. Wang X, Wang S, Lin YW, et al: Angiotensin-converting enzyme insertion/deletion polymorphism and the risk of prostate cancer in the Han population of China. Med Oncol 29: 1964-1971, 2012.

5. Giovannucci E, Stampfer MJ, Krithivas K, et al: The CAG repeat within the androgen receptor gene and its relationship to prostate cancer. Proc Natl Acad Sci USA 94: 3320-3323, 1997.

6. Merrill RM and Lyon JL: Explaining the difference in prostate cancer mortality rates between white and black men in the United States. Urology 55: 730-735, 2000

7. Souiden Y, Mahdouani M, Chaieb K, Elkamel R and Mahdouani K: Polymorphisms of glutathione-S-transferase M1 and $\mathrm{T} 1$ and prostate cancer risk in a Tunisian population. Cancer Epidemiol 34: 598-603, 2010.

8. McDonald AC, Jenkins FJ, Bunker CH, Wilson JW, Patrick AL and Weissfeld JL: A case-cohort study of human herpesvirus 8 seropositivity and incident prostate cancer in Tobago. Infect Agent Cancer 6: 25, 2011.

9. Hoffman LJ, Bunker CH, Pellett PE, et al: Elevated seroprevalence of human herpesvirus 8 among men with prostate cancer. J Infect Dis 189: 15-20, 2004.

10. Hrbacek J, Urban M, Hamsikova E, et al: Serum antibodies against genitourinary infectious agents in prostate cancer and benign prostate hyperplasia patients: a case-control study. BMC Cancer 11: 53, 2011.

11. Huang WY, Hayes R, Pfeiffer R, et al: Sexually transmissible infections and prostate cancer risk. Cancer Epidemiol Biomarkers Prev 17: 2374-2381, 2008.

12. Ewald PW: An evolutionary perspective on parasitism as a cause of cancer. Adv Parasitol 68: 21-43, 2009.

13. Haid M and Sharon N: Immunofluorescent evidence of prior herpes simplex virus type-2 infection in prostate carcinoma. Urology 24: 623-625, 1984

14. Dennis LK, Coughlin JA, McKinnon BC, et al: Sexually transmitted infections and prostate cancer among men in the U.S military. Cancer Epidemiol Biomarkers Prev 18: 2665-2671, 2009.

15. Korodi Z, Wang X, Tedeschi R, Knekt P and Dillner J: No serological evidence of association between prostate cancer and infection with herpes simplex virus type 2 or human herpesvirus type 8: a nested case-control study. J Infect Dis 191: 2008-2011, 2005 .

16. Jenkins FJ, Hayes RB, Jackson A, et al: Human herpesvirus 8 seroprevalence among prostate cancer case patients and control subjects. J Infect Dis 196: 208-211, 2007.

17. Sutcliffe S, Giovannucci E, Gaydos CA, et al: Plasma antibodies against Chlamydia trachomatis, human papillomavirus, and human herpesvirus type 8 in relation to prostate cancer: a prospective study. Cancer Epidemiol Biomarkers Prev 16: $1573-1580,2007$.

18. DerSimonian R and Laird N: Meta-analysis in clinical trials. Control Clin Trials 7: 177-188, 1986.

19. Higgins JP, Thompson SG, Deeks JJ and Altman DG: Measuring inconsistency in meta-analyses. BMJ 327: 557-560, 2003.

20. Higgins JP and Thompson SG: Quantifying heterogeneity in a meta-analysis. Stat Med 21: 1539-1558, 2002

21. Mantel N and Haenszel W: Statistical aspects of the analysis of data from retrospective studies of disease. J Natl Cancer Inst 22: 719-748, 1959

22. Fleiss JL: The statistical basis of meta-analysis. Stat Methods Med Res 2: 121-145, 1993.
23. Egger M, Davey Smith G, Schneider M and Minder C: Bias in meta-analysis detected by a simple, graphical test. BMJ 315 : 629-634, 1997.

24. Begg CB and Mazumdar M: Operating characteristics of a rank correlation test for publication bias. Biometrics 50: 1088-1101, 1994.

25. Herbert JT, Birkhoff JD, Feorino PM and Caldwell GG: Herpes simplex virus type 2 and cancer of the prostate. J Urol 116: 611-612, 1976.

26. Baker LH, Mebust WK, Chin TD, Chapman AL, Hinthorn D and Towle D: The relationship of herpesvirus to carcinoma of the prostate. J Urol 125: 370-374, 1981.

27. Luleci G, Sakizli M, Gunalp A, Erkan I and Remzi D: Herpes simplex type 2 neutralization antibodies in patients with cancers of urinary bladder, prostate, and cervix. J Surg Oncol 16: 327-331, 1981.

28. Siracusano F, Tarro G and Biviano D: TAF-test: a tumor diagnosis device in oncologic urology. Cancer 50: 2215-2217, 1982.

29. Csata S, Dan P, Kulcsar G, Horvath J, Nasz I and Verebelyi A: Viral examinations in malignant tumours of the urogenital system. Acta Chir Hung 25: 201-205, 1984.

30. Mandel JS and Schuman LM: Sexual factors and prostatic cancer: results from a case-control study. J Gerontol 42: 259-264, 1987.

31. Sabin AB and Tarro G: Herpes simplex and herpes genitalis viruses in etiology of some human cancers. Proc Natl Acad Sci USA 70: 3225-3229, 1973.

32. Coussens LM and Werb Z: Inflammation and cancer. Nature 420: 860-867, 2002

33. De Marzo AM, Platz EA, Sutcliffe S, et al: Inflammation in prostate carcinogenesis. Nat Rev Cancer 7: 256-269, 2007.

34. Palapattu GS, Sutcliffe S, Bastian PJ, et al: Prostate carcinogenesis and inflammation: emerging insights. Carcinogenesis 26: 1170-1181, 2005

35. Platz EA and De Marzo AM: Epidemiology of inflammation and prostate cancer. J Urol 171: S36-S40, 2004.

36. Kundu SD, Lee C, Billips BK, et al: The toll-like receptor pathway: a novel mechanism of infection-induced carcinogenesis of prostate epithelial cells. Prostate 68: 223-229, 2008.

37. Smith JS and Robinson NJ: Age-specific prevalence of infection with herpes simplex virus types 2 and 1: a global review. J Infect Dis 186 (Suppl 1): S3-S28, 2002.

38. National Cancer Institute: Howlader N, Noone AM, Krapcho M, et al: SEER Cancer Statistics Review, 1975-2009 (Vintage 2009 Populations). http://seer.cancer.gov/csr/1975_2009_pops09/. Accessed July 22,2012.

39. Diamond C, Brodie SJ, Krieger JN, et al: Human herpesvirus 8 in the prostate glands of men with Kaposi's sarcoma. J Virol 72: 6223-6227, 1998

40. Staskus KA, Zhong W, Gebhard K, et al: Kaposi's sarcoma-associated herpesvirus gene expression in endothelial (spindle) tumor cells. J Virol 71: 715-719, 1997.

41. Monini P, de Lellis L, Fabris M, Rigolin F and Cassai E: Kaposi's sarcoma-associated herpesvirus DNA sequences in prostate tissue and human semen. N Engl J Med 334: 1168-1172, 1996.

42. Rubin MA, Parry JP and Singh B: Kaposi's sarcoma associated herpesvirus deoxyribonucleic acid sequences: lack of detection in prostatic tissue of human immunodeficiency virus-negative immunocompetent adults. J Urol 159: 146-148, 1998.

43. Diamond C, Huang ML, Kedes DH, et al: Absence of detectable human herpesvirus 8 in the semen of human immunodeficiency virus-infected men without Kaposi's sarcoma. J Infect Dis 176: 775-777, 1997.

44. Corbellino M, Bestetti G, Galli M and Parravicini C: Absence of HHV-8 in prostate and semen. N Engl J Med 335: 1237; author reply $1238-1239,1996$.

45. Tasaka T, Said JW, Morosetti R, et al: Is Kaposi's sarcoma - associated herpesvirus ubiquitous in urogenital and prostate tissues? Blood 89: 1686-1689, 1997.

46. Moore PS and Chang Y: Kaposi's sarcoma-associated herpesvirus. In: Fields' Virology. 2nd edition. Knipe DM, Howley PM, Griffin DE, Lamb RA, Martin MA, Roizman B and Straus SE (eds). Lippincott Williams \& Wilkins, Philadelphia, pp2803-2833, 2001.

47. Boldogh I, Baskar JF, Mar EC and Huang ES: Human cytomegalovirus and herpes simplex type 2 virus in normal and adenocarcinomatous prostate glands. J Natl Cancer Inst 70: 819-826, 1983. 